\title{
A CLOSE ASSOCIATION OF HEPATOCELLULAR CARCINOMA WITH CIRRHOTIC CHANGES AMONG 275, 857 PATHOLOGICAL AUTOPSY CASES IN JAPAN DURING 16YEARS FROM 1958 TO 1973
}

\author{
TORU MIYAJI \\ Japanese Institute of Patho'ogy, Osaka, 530, and Ciba-Geigy, \\ Takarazuka, 665, Japan
}

(Received for publication February 1, 1977)

\begin{abstract}
Based on the registered autopsy cases since 1958, the association of hepatocellular carcinoma with cirrhotic changes was studied on the 275,857 autopsy cases carried out in Japan during 16 years from 1958 to 1973.

1. The incidence of hepatocellular carcinoma among autopsies has been fairly high compared with Western countries, amounting 2.5 percent (3.5 percent in male and 1.2 percent in female).

2 . The incidence of cirrhotic changes among these autopsies was 6.3 percent in male and 4.3 percent in female. Presumably, the majority of cirrhosis in Japan is of multilobular thin septal pattern, which has been popularly called as B type cirrhosis of Nagayo-Miyake's classification or posthepatitic type of Gall's classification.

3 . The association of hepatocellular carcinoma with cirrhotic changes is found as 70.0 percent in male and 53.3 percent in female $(66.8$ percent in total). On the other hand, cirrhotic changes, particularly those above mentioned, are closely associated with hepatocellular carcinoma, and it was found that 23.3 percent of cirrhotic changes (27.2 percent in male and 12.9 percent in female) were associated with hepatocellular carcinoma.

4. Cholangiocellular carcinoma is also asociated with cirrhosis to the less extent than hepatocellular carcinoma (20.7 percent in male and 10.7 percent in female), and cirrhotic changes are not associated with cholangiocellular carcinoma.

5. Age incidences of hepatocellular carcinoma, B type cirrhosis and cirrhosis NOS are presented. The peaks are at 55 years old, 60 and 60 respectively.
\end{abstract}

\section{INTRODUCTION}

Based on scanty autopsy cases up to 1940 in Japan, Berman (Berman, 1951) included Japan among the countries showing suspected high incidence of liver cancer in 1950. This high incidence, however, has been repeatedly confirmed by many pathologists in Japan although their autopsy numbers were comparatively limited (Miyaji et al., 1960 ; Miyaji, 1965 ; Miyaji, 1970), Segi and Kurihara (Segi and Kurihara, 1972) have also repeatedly described the high incidence of hepatic malignancies in Japan among 24 countries based on the 
cancer mortality. As the matter of fact, the Japanese holds the highest incidence in males and the third in females among these countries. The present report deals with a close association of hepatocellular carcinoma with cirrhotic changes in Japan based on registered autopsy cases during 16 years from 1958 to 1973 inclusive.

\section{MATERIAL AND METHOD}

Since 1958, the Annual of the Pathological Autopsy Cases in Japan has been published as a registry of autopsies, and it covers more than 95 percent of the pathological autopsies in Japan each year. From 16 volumes of the Annuals published by the Japanese Pathological Society from 1958 to 1973 each year, every registered cases was recorded on an IBM-type card following a disease code comprising 1,036 pathological findings in addition to age, sex, year of autopsy, source and autopsy number. The cards were counted and assorted with a mark system selector (Tanac).

\section{RESULTS}

\section{Frequent malignancies among autopsy cases}

Six years' autopsy cases from 1968 to 1973 inclusive, 82,871 males and 55,953 females altogether, revealed that the most frequent malignancies were gastric carcinoma followed by leukemia, bronchogenic carcinoma, hepatecellular carcinoma and so on as shown in Table 1. Fig. 1 shows the sites of these malignancies according to sex, and cervical carcinoma was added. As described by Segi and Kurihara (Segi and Kurihara, 1972), malignant neoplasm of the stomach in Japan holds the highest in-
TABLE 1

Frequent malignancies among autopsies in Japan during 6 years from 1968 to 1973

\begin{tabular}{ll|c}
\hline \multicolumn{1}{c|}{ Malignancies } & Total number \\
\hline 1 & Stomach & 20,253 \\
2 & Leukemia & 9,786 \\
3 & Lung & 9,149 \\
4 & Liver & 4,178 \\
5 & Malignant lymphoma & 3,272 \\
6 & Pancreas & 3,151 \\
7 & Esophagus & 2,140 \\
8 & Colon & 1,770 \\
9 & Gallbladder & 1,517 \\
10 & Rectum & 1,480 \\
11 & Extrahepatic bile duct & 1,361 \\
\hline & Uterine cervix & 2,341 \\
\hline
\end{tabular}

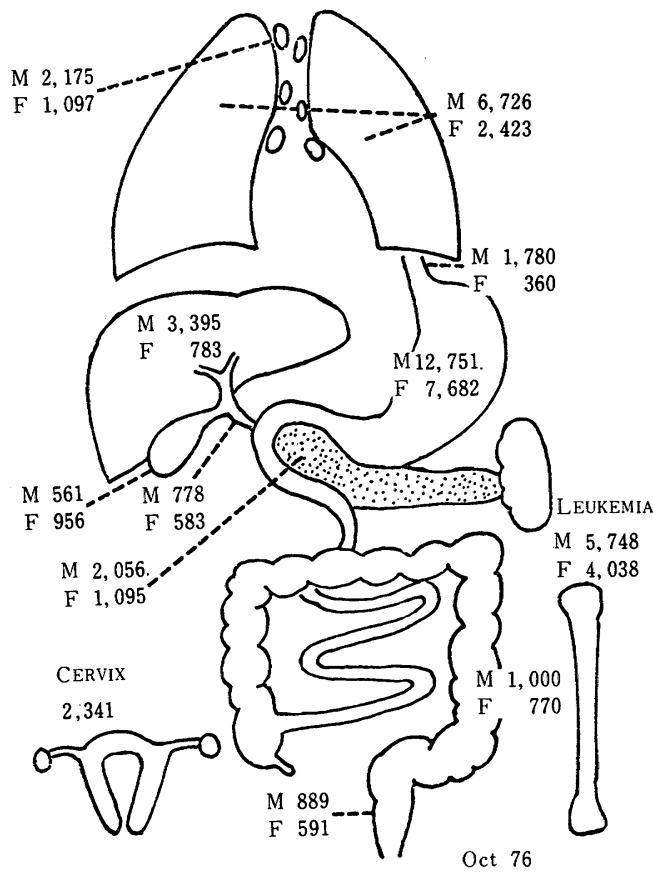

Fig. 1 Frequent malignancies among autopsies in Japan during 6 years from 1968 to 1973. 
cidence among 24 countires, and has been the most frequent one in the Vital Statistics in Japan. These are also reflected on the autopsy cases, and it is noteworthy that the hepatocellular carcinoma ranks the fourth among the frequent malignancies.

2. Number of total autopsy cases, hepatocellular carcinoma and cirrhosis in comparison with those in the Vital Statistics

During 16 years from 1958 to 1973, the number of autopsy cases registered were 275,857 in total as shown in Table 2. The number of autopsies reached the peak with 25,407 a year in 1968, and then decreased in 1969 when student riots started at universities almost all over Japan. The number of sex-unkno-

TABLE 2

Number of autopsy cases in Japan during 16 years from 1958 to 1973

\begin{tabular}{r|r|r|r|r}
\hline Year & Male & Female & $\begin{array}{r}\text { Sex } \\
\text { unk. }\end{array}$ & Total \\
\hline 1958 & 5,231 & 3,233 & 150 & 8,614 \\
1959 & 5,275 & 3,571 & 180 & 9,026 \\
1960 & 5,933 & 3,770 & 202 & 9,905 \\
1961 & 6,951 & 4,495 & 206 & 11,296 \\
1962 & 7,311 & 5,074 & 214 & 12,599 \\
1963 & 8,407 & 5,495 & 195 & 14,061 \\
1964 & 8,900 & 5,831 & 245 & 14,976 \\
1965 & 10,283 & 6,565 & 170 & 17,018 \\
1966 & 10,657 & 7,222 & 47 & 17,926 \\
1967 & 12,558 & 8,395 & 97 & 21,050 \\
1968 & 15,138 & 10,199 & 70 & 25,407 \\
1969 & 14,202 & 9,638 & 64 & 23,904 \\
1970 & 13,034 & 8,842 & 83 & 21,959 \\
1971 & 13,031 & 8,750 & 123 & 21,904 \\
1972 & 13,568 & 9,057 & 91 & 22,716 \\
1973 & 13,898 & 9,467 & 131 & 23,496 \\
\hline Total & 164,017 & 109,572 & 2,268 & 275,857 \\
\hline
\end{tabular}

Based on the Annuals of Pathological Auotpsy Cases in Japan wn cases decreased sharply since 1966 as the author made inquiry whenever sex-unknown or cases of ambiguous pathological findings were found, although the majority of sex-unknown were stillbirths and newborns amounting 33 and 24 percent respectively.

During these 16 years, 7,002 hepatocellular carcinomas and 15,136 cirrhotic changes which were not associated with hepatocellular carcnoma nor cholangiocellular carcinoma were found among autopsies as shown in Tables 3 and 8 . These cases were enumerated as such irrespective of lesions of the liver which were described as manifest or latent, and primary or accessory. It did show a gradual increase in number of hepatocellular carcinoma with year more marked in male than in female.

On the other hand, total deaths and

\section{TABLE 3}

Total number of autopsied hepatocellular carcinoma in Japan (1958-73)

\begin{tabular}{c|c|c|c}
\hline Year & Male & Female & Sex unk. \\
\hline 1958 & 118 & 30 & 1 \\
1959 & 126 & 30 & 0 \\
1960 & 149 & 43 & 0 \\
1961 & 176 & 52 & 0 \\
1962 & 194 & 30 & 0 \\
1963 & 221 & 45 & 1 \\
1964 & 261 & 76 & 2 \\
1965 & 288 & 60 & 0 \\
1966 & 346 & 62 & 0 \\
1967 & 386 & 97 & 5 \\
1968 & 545 & 116 & 0 \\
1969 & 510 & 117 & 0 \\
1970 & 543 & 113 & 0 \\
1971 & 584 & 136 & 0 \\
1972 & 645 & 139 & 2 \\
1973 & 568 & 162 & 0 \\
\hline Total & 5,660 & 1,331 & 11 \\
\hline
\end{tabular}


deaths due to "malignant primary neoplasm of the liver (ICD 155.0)" and "cirrhosis of the liver (ICD 571)" obtained from the Vital Statistics of the Ministry of Health and Welfare during the same 16 years are shown in Tables 4,5 and 6 . It is clear that only 2.75 percent of male and 2.16 percent of female deaths were autopsied and registered in the Annual of Pathological Autopsy Cases during these 16 years. Assuming that all the clinical diagnoses of hepatocellular carcinoma and cirrhosis were correct as these were listed in the Vital Statistics, it appears as if patients with hepatocellular carcinoma

\section{TABLE 4}

Total number of deaths listed in vital statistics and autopsies in Japan during 16 years from 1958 to 1973

\begin{tabular}{l|r|r}
\hline & \multicolumn{1}{|c|}{ Male } & \multicolumn{1}{|c}{ Female } \\
\hline Deaths & $5,964,251$ & $5,082,993$ \\
Autopsies & 164,017 & 109,572 \\
Ratio & $2.75 \%$ & $2.16 \%$ \\
\hline
\end{tabular}

TABLE 5

Number of hepatocellular carcinoma among vital statistics and autopsies in Japan during 16 years from 1958 to 1973

\begin{tabular}{l|r|r}
\hline & Male & Female \\
\hline Deaths & 10,978 & 6,726 \\
Autopsies & 5,660 & 1,331 \\
Ratio & $51.56 \%$ & $19.79 \%$ \\
\hline
\end{tabular}

\section{TABLE 6}

Number of cirrhosis among vital statistics and autopsies in Japan during 16 years from 1958 to 1973

\begin{tabular}{l|r|r}
\hline & Male & Female \\
\hline Deaths & 114,600 & 57,368 \\
Autopsies & 10,395 & 4,741 \\
Ratio & $9.07 \%$ & $8.26 \%$ \\
\hline
\end{tabular}

were autopsied in the ratio of 51.6 percent in male and 19.8 percent in female, and patients with cirrhosis 9.1 percent in male and 8.3 percent in female. These marked discrepancies between autopsy cases and the Vital Statistics are not easily explained, although it is well known that autopsy cases are biased and patients with severe diseases such as hepatocellular carcinoma or cirrhosis are likely to be treated at large and well-organized hospitals, and autopsied when they pass away.

The above figures among autopsy cases do also include hepatocellular carcinoma as well as cirrhosis, which were not recognized or diagnosed clinically.

As it will be mentioned later, most of hepatocellular carcinoma in Japan are associated with cirrhosis, and are liable to be covered by clinical symptoms of cirrhosis. In other words, hepatocellular carcinoma are often underdiagnosed, particularly when they are associated with cirrhosis, although the determination of alpha-fetoprotein has made the diagnosis more accurate in recent years (Hirai, 1975). Consequently, it was considered that the number of deaths due to hepatocellular carcinoma would have been higher than that appeared in the Vital Statistics.

3. Association of hepatocellular carcinoma and cholangiocellular carcinoma with cirrhosis

Since Nagayo in 1914 (Nagayo, 1914), a morphological classification of cirrhosis based on the width of septum surrounding pseudolobules has been carried out in Japan. Wide septal cirrhosis as shown in Photo 1 was called as A type cirrhosis, and narrow septal cirrhosis as shown in Photo 2 as B type. The transitional type between $A$ and $B$ type was named as $A^{\prime}$ type cirrhosis. 


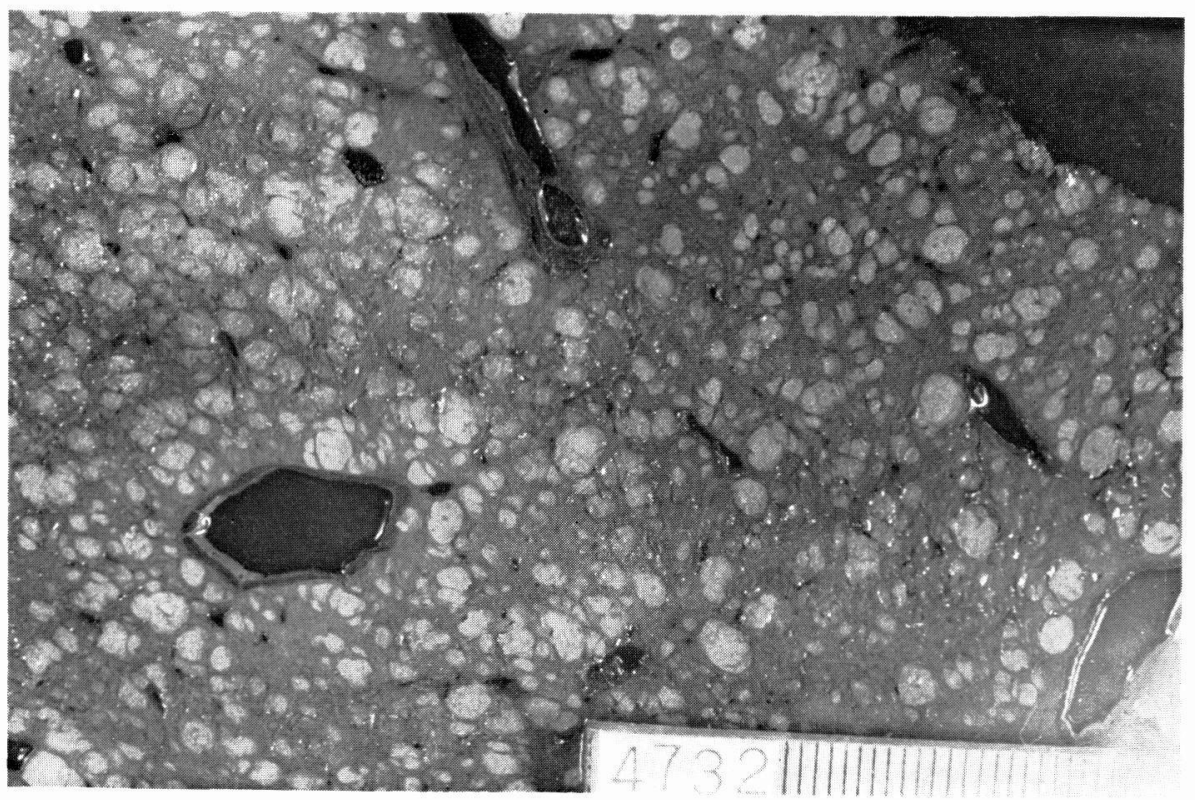

Photo 1 A type cirrhosis of Nagayo-Miyake's classification (postnecrotic cirrhosis): Pseudolobules are surrounded by wide septa with fibrosis and cellular infiltration of mainly mononuclear cells.

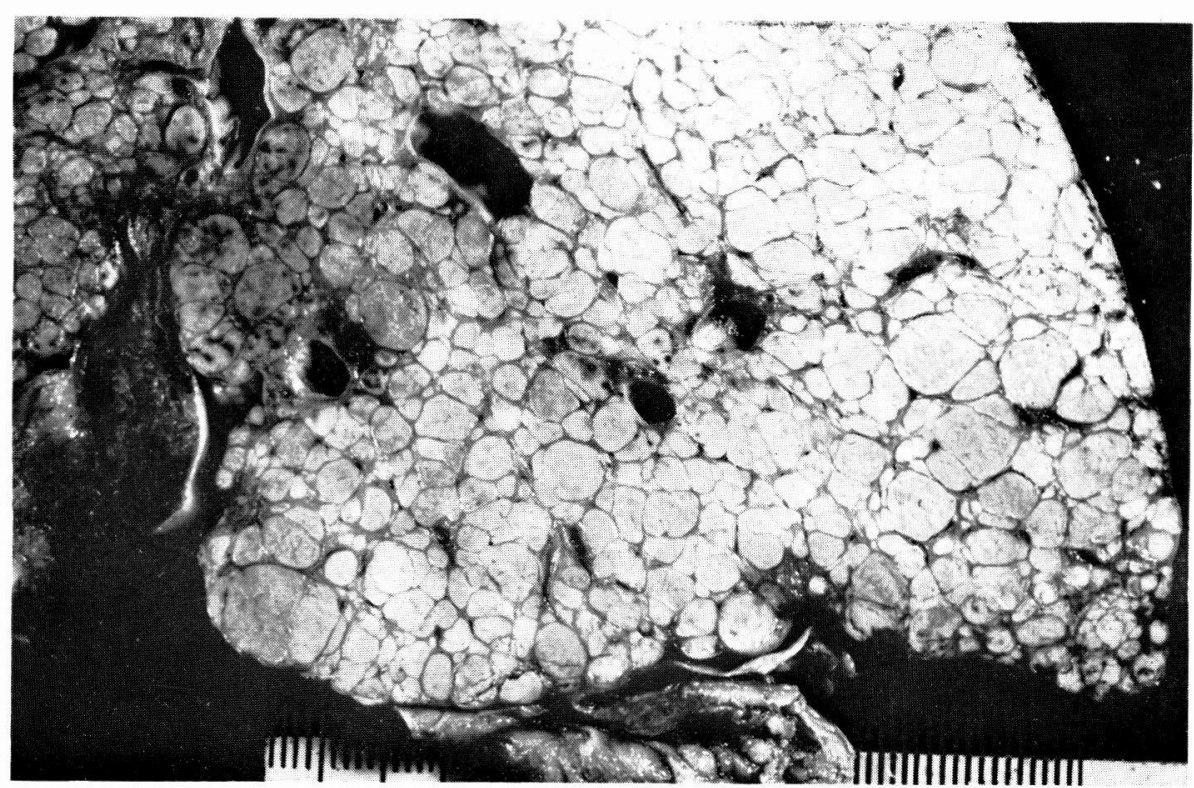

Photo 2 B type cirrhosis of Nagayo-Miyake's classification (posthepatic or macronodular cirrhosis): Large or multilobular pseudolobules are surrounded by thin septa, and this is the predominant cirrhosis most frequently associated with hepatocellular carcinoma in Japan. 
Miyake (Miyake, 1960) added later a transitional type between fibrosis and B type cirrhosis, in which the septum surrounding pseudolobules is not completed, and named it as B' type cirrhosis. Although these classifications do not identify whether it is macronodular, micronodular or mixed, they do correspond well with popular classifications such as Gall's as shown in Table 7.
TABLE 7

Correspondence of Nagayo-Miyake's to Gall's Classification of cirrhosis

\begin{tabular}{ll}
\hline Nagayo-Miyake & Gall \\
\hline B type & Posthepatitic \\
Fatty cirrhosis* & Nutritional \\
A type & Postnecrotic \\
A' type & Mixed \\
\hline
\end{tabular}

* Without fatty change, could be classified as B type.

TABLE 8

Fibrosis and cirrhosis associated with hepatocellular carcinoma among autopsy cases in Japan during 16 years from 1958 to 1973

\begin{tabular}{|c|c|c|c|c|c|c|c|c|c|c|c|}
\hline Year & Sex & Totl & Fibr & Fat C & Bil C & Card & A typ & $A^{\prime}$ typ & B typ & $\mathrm{C}$ NOS & $B^{\prime}$ typ \\
\hline 1958 & $\frac{\mathrm{M}}{\mathrm{F}}$ & $\begin{array}{r}118 \\
30\end{array}$ & $\begin{array}{l}0 \\
0\end{array}$ & $\begin{array}{l}0 \\
0\end{array}$ & $\begin{array}{l}1 \\
0\end{array}$ & $\begin{array}{l}0 \\
0\end{array}$ & $\begin{array}{l}1 \\
0\end{array}$ & $\begin{array}{l}0 \\
0\end{array}$ & $\begin{array}{r}24 \\
0\end{array}$ & $\begin{array}{r}44 \\
1\end{array}$ & $\begin{array}{l}0 \\
0\end{array}$ \\
\hline 1959 & $\frac{\mathrm{M}}{\mathrm{F}}$ & $\begin{array}{r}126 \\
30\end{array}$ & $\begin{array}{l}3 \\
1\end{array}$ & $\begin{array}{l}0 \\
0\end{array}$ & $\begin{array}{l}1 \\
0\end{array}$ & $\begin{array}{l}0 \\
0\end{array}$ & $\begin{array}{l}0 \\
0\end{array}$ & $\begin{array}{l}0 \\
0\end{array}$ & $\begin{array}{r}27 \\
3\end{array}$ & $\begin{array}{r}29 \\
7\end{array}$ & $\begin{array}{l}1 \\
1\end{array}$ \\
\hline 1960 & $\frac{\mathrm{M}}{\mathrm{F}}$ & $\begin{array}{r}149 \\
43\end{array}$ & $\begin{array}{l}1 \\
0\end{array}$ & $\begin{array}{l}0 \\
0\end{array}$ & $\begin{array}{l}0 \\
0\end{array}$ & $\begin{array}{l}0 \\
0\end{array}$ & $\begin{array}{l}3 \\
0\end{array}$ & $\begin{array}{l}0 \\
0\end{array}$ & $\begin{array}{r}29 \\
5\end{array}$ & $\begin{array}{l}61 \\
13\end{array}$ & $\begin{array}{l}0 \\
0\end{array}$ \\
\hline 1961 & $\frac{M}{F}$ & $\begin{array}{r}176 \\
52\end{array}$ & $\begin{array}{l}1 \\
2\end{array}$ & $\begin{array}{l}0 \\
0\end{array}$ & $\begin{array}{l}0 \\
1\end{array}$ & $\begin{array}{l}1 \\
0\end{array}$ & $\begin{array}{l}6 \\
1\end{array}$ & $\begin{array}{l}0 \\
0\end{array}$ & $\begin{array}{l}56 \\
12\end{array}$ & $\begin{array}{l}54 \\
15\end{array}$ & $\begin{array}{l}1 \\
0\end{array}$ \\
\hline 1962 & $\frac{\mathrm{M}}{\mathrm{F}}$ & $\begin{array}{r}194 \\
30\end{array}$ & $\begin{array}{l}2 \\
0\end{array}$ & $\begin{array}{l}0 \\
0\end{array}$ & $\begin{array}{l}1 \\
0\end{array}$ & $\begin{array}{l}0 \\
1\end{array}$ & $\begin{array}{l}5 \\
0\end{array}$ & $\begin{array}{l}0 \\
0\end{array}$ & $\begin{array}{l}42 \\
11\end{array}$ & $\begin{array}{r}89 \\
4\end{array}$ & $\begin{array}{l}2 \\
0\end{array}$ \\
\hline 1963 & $\frac{\mathrm{M}}{\mathrm{F}}$ & $\begin{array}{r}221 \\
45\end{array}$ & $\begin{array}{l}0 \\
1\end{array}$ & $\begin{array}{l}0 \\
0\end{array}$ & $\begin{array}{l}1 \\
0\end{array}$ & $\begin{array}{l}1 \\
1\end{array}$ & $\begin{array}{r}13 \\
3\end{array}$ & $\begin{array}{l}0 \\
1\end{array}$ & $\begin{array}{r}57 \\
1\end{array}$ & $\begin{array}{l}72 \\
12\end{array}$ & $\begin{array}{l}2 \\
1\end{array}$ \\
\hline 1964 & $\frac{\mathrm{M}}{\mathrm{F}}$ & $\begin{array}{r}261 \\
76\end{array}$ & $\begin{array}{l}0 \\
2\end{array}$ & $\begin{array}{l}0 \\
0\end{array}$ & $\begin{array}{l}2 \\
1\end{array}$ & $\begin{array}{l}0 \\
0\end{array}$ & $\begin{array}{r}21 \\
3\end{array}$ & $\begin{array}{l}0 \\
1\end{array}$ & $\begin{array}{l}64 \\
14\end{array}$ & $\begin{array}{l}87 \\
18\end{array}$ & $\begin{array}{l}0 \\
2\end{array}$ \\
\hline 1965 & $\frac{\mathrm{M}}{\mathrm{F}}$ & $\begin{array}{r}288 \\
62\end{array}$ & $\begin{array}{l}6 \\
0\end{array}$ & $\begin{array}{l}0 \\
0\end{array}$ & $\begin{array}{l}2 \\
0\end{array}$ & $\begin{array}{l}1 \\
0\end{array}$ & $\begin{array}{r}14 \\
2\end{array}$ & $\begin{array}{l}0 \\
0\end{array}$ & $\begin{array}{l}53 \\
12\end{array}$ & $\begin{array}{r}110 \\
18\end{array}$ & $\begin{array}{l}1 \\
1\end{array}$ \\
\hline 1966 & $\frac{\mathrm{M}}{\mathrm{F}}$ & $\begin{array}{r}346 \\
83\end{array}$ & $\begin{array}{l}6 \\
1\end{array}$ & $\begin{array}{l}0 \\
0\end{array}$ & $\begin{array}{l}0 \\
1\end{array}$ & $\begin{array}{l}0 \\
1\end{array}$ & $\begin{array}{r}18 \\
3\end{array}$ & $\begin{array}{l}3 \\
2\end{array}$ & $\begin{array}{l}48 \\
12\end{array}$ & $\begin{array}{r}117 \\
19\end{array}$ & $\begin{array}{l}5 \\
1\end{array}$ \\
\hline 1967 & $\frac{\mathrm{M}}{\mathrm{F}}$ & $\begin{array}{r}386 \\
97\end{array}$ & $\begin{array}{l}3 \\
2\end{array}$ & $\begin{array}{l}0 \\
0\end{array}$ & $\begin{array}{l}1 \\
0\end{array}$ & $\begin{array}{l}1 \\
0\end{array}$ & $\begin{array}{r}20 \\
3\end{array}$ & $\begin{array}{l}6 \\
2\end{array}$ & $\begin{array}{r}101 \\
16\end{array}$ & $\begin{array}{r}132 \\
24\end{array}$ & $\begin{array}{r}11 \\
1\end{array}$ \\
\hline 1968 & $\frac{\mathrm{M}}{\mathrm{F}}$ & $\begin{array}{l}545 \\
116\end{array}$ & $\begin{array}{l}8 \\
0\end{array}$ & $\begin{array}{l}1 \\
0\end{array}$ & $\begin{array}{l}2 \\
1\end{array}$ & $\begin{array}{l}1 \\
0\end{array}$ & $\begin{array}{r}23 \\
2\end{array}$ & $\begin{array}{r}13 \\
0\end{array}$ & $\begin{array}{r}164 \\
37\end{array}$ & $\begin{array}{r}173 \\
26\end{array}$ & $\begin{array}{l}9 \\
0\end{array}$ \\
\hline 1969 & $\frac{\mathrm{M}}{\mathrm{F}}$ & $\begin{array}{l}510 \\
117\end{array}$ & $\begin{array}{l}6 \\
2\end{array}$ & $\begin{array}{l}0 \\
0\end{array}$ & $\begin{array}{l}1 \\
2\end{array}$ & $\begin{array}{l}1 \\
1\end{array}$ & $\begin{array}{r}25 \\
7\end{array}$ & $\begin{array}{l}0 \\
0\end{array}$ & $\begin{array}{r}137 \\
18\end{array}$ & $\begin{array}{r}201 \\
28\end{array}$ & $\begin{array}{l}1 \\
0\end{array}$ \\
\hline 1970 & $\frac{\mathrm{M}}{\mathrm{F}}$ & $\begin{array}{l}543 \\
113\end{array}$ & $\begin{array}{l}1 \\
0\end{array}$ & $\begin{array}{l}1 \\
0\end{array}$ & $\begin{array}{l}3 \\
0\end{array}$ & $\begin{array}{l}0 \\
1\end{array}$ & $\begin{array}{l}37 \\
12\end{array}$ & $\begin{array}{l}1 \\
1\end{array}$ & $\begin{array}{r}168 \\
24\end{array}$ & $\begin{array}{r}167 \\
24\end{array}$ & $\begin{array}{l}0 \\
0\end{array}$ \\
\hline 1971 & $\frac{\mathrm{M}}{\mathrm{F}}$ & $\begin{array}{l}584 \\
136\end{array}$ & $\begin{array}{l}6 \\
0\end{array}$ & $\begin{array}{l}1 \\
0\end{array}$ & $\begin{array}{l}1 \\
0\end{array}$ & $\begin{array}{l}1 \\
0\end{array}$ & $\begin{array}{r}37 \\
8\end{array}$ & $\begin{array}{r}16 \\
2\end{array}$ & $\begin{array}{r}228 \\
32\end{array}$ & $\begin{array}{r}142 \\
23\end{array}$ & $\begin{array}{l}9 \\
3\end{array}$ \\
\hline 1972 & $\frac{\mathrm{M}}{\mathrm{F}}$ & $\begin{array}{l}645 \\
139\end{array}$ & $\begin{array}{r}12 \\
3\end{array}$ & $\begin{array}{l}0 \\
0\end{array}$ & $\begin{array}{l}3 \\
1\end{array}$ & $\begin{array}{l}0 \\
0\end{array}$ & $\begin{array}{l}55 \\
15\end{array}$ & $\begin{array}{r}23 \\
6\end{array}$ & $\begin{array}{r}285 \\
51\end{array}$ & $\begin{array}{r}102 \\
13\end{array}$ & $\begin{array}{l}8 \\
4\end{array}$ \\
\hline 1973 & $\begin{array}{l}\mathrm{M} \\
\mathrm{F}\end{array}$ & $\begin{array}{l}568 \\
162 \\
\end{array}$ & $\begin{array}{l}9 \\
0 \\
\end{array}$ & $\begin{array}{l}1 \\
0 \\
\end{array}$ & $\begin{array}{l}2 \\
0 \\
\end{array}$ & $\begin{array}{l}1 \\
0 \\
\end{array}$ & $\begin{array}{l}45 \\
13 \\
\end{array}$ & $\begin{array}{r}19 \\
4\end{array}$ & $\begin{array}{r}226 \\
56 \\
\end{array}$ & $\begin{array}{l}70 \\
14 \\
\end{array}$ & $\begin{array}{r}15 \\
6 \\
\end{array}$ \\
\hline Total & $\frac{\mathrm{M}}{\mathrm{F}}$ & $\begin{array}{l}5,660 \\
1,331\end{array}$ & $\begin{array}{l}64 \\
14\end{array}$ & $\begin{array}{l}4 \\
0\end{array}$ & $\begin{array}{r}21 \\
7\end{array}$ & $\begin{array}{l}8 \\
4\end{array}$ & $\begin{array}{r}323 \\
72\end{array}$ & $\begin{array}{l}81 \\
19\end{array}$ & $\begin{array}{r}1,745 \\
305\end{array}$ & $\begin{array}{r}1,650 \\
268\end{array}$ & $\begin{array}{l}65 \\
20\end{array}$ \\
\hline
\end{tabular}

Fibr=fibrosis, Fat $\mathrm{C}=$ fatty cirrhosis, Bil $\mathrm{C}=$ biliary cirrhosis, Card=cardiac cirrhosis, A typ $=$ A type cirrhosis, B typ $=$ B type cirrhosis, C NOS $=$ cirrhosis NOS 
Based on the above-mentioned $\mathrm{Na}$ gayo-Miyake's classification of cirrhosis, various types of cirrhoses associated with hepatocellular carcinoma, cholangiocellular carcinoma and those not associated with these two malignancies found among registered autopsies during 16 years are shown in Tables 8,9 and 10. Other cirrhotic changes of the liver, such as Wilson's disease, hemochromatosis, Budd-Chiari's disease, membranous obliteration of inferior vena cava in the hepatic part and primary biliary cirrhosis are not listed as these lesions were very small in number and rarely associated with hepatocellular and cholangiocellular carcinoma.

Tables 11 and 12 show that 70.0 percent of hepatocellular carcinoma in male and 53.3 percent in female are associated with cirrhosis. As a control of hepatocellular carcinoma, the assocation

TABLE 9

Fibrosis and cirrhosis associated with intrahepatic cholangiocarcinoma among autopsies (1958 - 1973)

\begin{tabular}{|c|c|c|c|c|c|c|c|c|c|c|c|}
\hline Year & Sex & Totl & Fibr & Fat C & Bil C & Card & A typ & $A^{\prime}$ typ & B typ & C NOS & $B^{\prime}$ typ \\
\hline 1958 & $\frac{\mathrm{M}}{\mathrm{F}}$ & $\begin{array}{l}30 \\
18\end{array}$ & $\begin{array}{l}0 \\
0\end{array}$ & $\begin{array}{l}0 \\
0\end{array}$ & $\begin{array}{l}0 \\
0\end{array}$ & $\begin{array}{l}0 \\
0\end{array}$ & $\begin{array}{l}0 \\
0\end{array}$ & $\begin{array}{l}0 \\
0\end{array}$ & $\begin{array}{l}1 \\
0\end{array}$ & $\begin{array}{l}1 \\
0\end{array}$ & $\begin{array}{l}0 \\
0\end{array}$ \\
\hline 1959 & $\frac{\mathrm{M}}{\mathrm{F}}$ & $\begin{array}{l}21 \\
15\end{array}$ & $\begin{array}{l}0 \\
0\end{array}$ & $\begin{array}{l}0 \\
0\end{array}$ & $\begin{array}{l}1 \\
0\end{array}$ & $\begin{array}{l}0 \\
0\end{array}$ & $\begin{array}{l}0 \\
0\end{array}$ & $\begin{array}{l}0 \\
0\end{array}$ & $\begin{array}{l}0 \\
0\end{array}$ & $\begin{array}{l}2 \\
0\end{array}$ & $\begin{array}{l}0 \\
0\end{array}$ \\
\hline 1960 & $\frac{\mathrm{M}}{\mathrm{F}}$ & $\begin{array}{l}41 \\
13\end{array}$ & $\begin{array}{l}1 \\
0\end{array}$ & $\begin{array}{l}0 \\
0\end{array}$ & $\begin{array}{l}3 \\
0\end{array}$ & $\begin{array}{l}0 \\
0\end{array}$ & $\begin{array}{l}1 \\
0\end{array}$ & $\begin{array}{l}0 \\
0\end{array}$ & $\begin{array}{l}3 \\
0\end{array}$ & $\begin{array}{l}2 \\
0\end{array}$ & $\begin{array}{l}0 \\
0\end{array}$ \\
\hline 1961 & $\frac{\mathrm{M}}{\mathrm{F}}$ & $\begin{array}{l}26 \\
24\end{array}$ & $\begin{array}{l}1 \\
0\end{array}$ & $\begin{array}{l}0 \\
0\end{array}$ & $\begin{array}{l}0 \\
5\end{array}$ & $\begin{array}{l}0 \\
0\end{array}$ & $\begin{array}{l}1 \\
1\end{array}$ & $\begin{array}{l}0 \\
0\end{array}$ & $\begin{array}{l}0 \\
0\end{array}$ & $\begin{array}{l}3 \\
1\end{array}$ & $\begin{array}{l}2 \\
0\end{array}$ \\
\hline 1962 & $\frac{\mathrm{M}}{\mathrm{F}}$ & $\begin{array}{l}37 \\
20\end{array}$ & $\begin{array}{l}0 \\
0\end{array}$ & $\begin{array}{l}0 \\
0\end{array}$ & $\begin{array}{l}2 \\
0\end{array}$ & $\begin{array}{l}0 \\
0\end{array}$ & $\begin{array}{l}0 \\
0\end{array}$ & $\begin{array}{l}0 \\
0\end{array}$ & $\begin{array}{l}2 \\
1\end{array}$ & $\begin{array}{l}2 \\
2\end{array}$ & $\begin{array}{l}0 \\
0\end{array}$ \\
\hline 1963 & $\frac{\mathrm{M}}{\mathrm{F}}$ & $\begin{array}{l}31 \\
25\end{array}$ & $\begin{array}{l}0 \\
0\end{array}$ & $\begin{array}{l}0 \\
0\end{array}$ & $\begin{array}{l}1 \\
2\end{array}$ & $\begin{array}{l}0 \\
0\end{array}$ & $\begin{array}{l}1 \\
1\end{array}$ & $\begin{array}{l}0 \\
0\end{array}$ & $\begin{array}{l}1 \\
0\end{array}$ & $\begin{array}{l}3 \\
0\end{array}$ & $\begin{array}{l}1 \\
0\end{array}$ \\
\hline 1964 & $\frac{\mathrm{M}}{\mathrm{F}}$ & $\begin{array}{l}41 \\
21\end{array}$ & $\begin{array}{l}0 \\
0\end{array}$ & $\begin{array}{l}0 \\
0\end{array}$ & $\begin{array}{l}2 \\
1\end{array}$ & $\begin{array}{l}0 \\
0\end{array}$ & $\begin{array}{l}1 \\
1\end{array}$ & $\begin{array}{l}0 \\
0\end{array}$ & $\begin{array}{l}2 \\
1\end{array}$ & $\begin{array}{l}2 \\
1\end{array}$ & $\begin{array}{l}0 \\
0\end{array}$ \\
\hline 1965 & $\frac{\mathrm{M}}{\mathrm{F}}$ & $\begin{array}{l}63 \\
24\end{array}$ & $\begin{array}{l}1 \\
0\end{array}$ & $\begin{array}{l}0 \\
0\end{array}$ & $\begin{array}{l}7 \\
1\end{array}$ & $\begin{array}{l}0 \\
0\end{array}$ & $\begin{array}{l}1 \\
1\end{array}$ & $\begin{array}{l}0 \\
0\end{array}$ & $\begin{array}{l}2 \\
1\end{array}$ & $\begin{array}{l}5 \\
1\end{array}$ & $\begin{array}{l}0 \\
0\end{array}$ \\
\hline 1966 & $\frac{\mathrm{M}}{\mathrm{F}}$ & $\begin{array}{l}50 \\
33\end{array}$ & $\begin{array}{l}1 \\
1\end{array}$ & $\begin{array}{l}0 \\
0\end{array}$ & $\begin{array}{l}6 \\
0\end{array}$ & $\begin{array}{l}0 \\
1\end{array}$ & $\begin{array}{l}0 \\
0\end{array}$ & $\begin{array}{l}0 \\
0\end{array}$ & $\begin{array}{l}4 \\
0\end{array}$ & $\begin{array}{l}6 \\
2\end{array}$ & $\begin{array}{l}0 \\
0\end{array}$ \\
\hline 1967 & $\frac{\mathrm{M}}{\mathrm{F}}$ & $\begin{array}{l}94 \\
43\end{array}$ & $\begin{array}{l}3 \\
2\end{array}$ & $\begin{array}{l}0 \\
0\end{array}$ & $\begin{array}{r}13 \\
4\end{array}$ & $\begin{array}{l}0 \\
0\end{array}$ & $\begin{array}{l}0 \\
0\end{array}$ & $\begin{array}{l}0 \\
0\end{array}$ & $\begin{array}{l}4 \\
1\end{array}$ & $\begin{array}{l}4 \\
1\end{array}$ & $\begin{array}{l}0 \\
0\end{array}$ \\
\hline 1968 & $\frac{\mathrm{M}}{\mathrm{F}}$ & $\begin{array}{l}79 \\
54\end{array}$ & $\begin{array}{l}2 \\
6\end{array}$ & $\begin{array}{l}0 \\
0\end{array}$ & $\begin{array}{l}3 \\
1\end{array}$ & $\begin{array}{l}0 \\
0\end{array}$ & $\begin{array}{l}0 \\
1\end{array}$ & $\begin{array}{l}0 \\
0\end{array}$ & $\begin{array}{l}7 \\
0\end{array}$ & $\begin{array}{l}4 \\
0\end{array}$ & $\begin{array}{l}0 \\
0\end{array}$ \\
\hline 1969 & $\frac{\mathrm{M}}{\mathrm{F}}$ & $\begin{array}{l}68 \\
54\end{array}$ & $\begin{array}{l}0 \\
1\end{array}$ & $\begin{array}{l}0 \\
0\end{array}$ & $\begin{array}{l}4 \\
1\end{array}$ & $\begin{array}{l}0 \\
0\end{array}$ & $\begin{array}{l}2 \\
0\end{array}$ & $\begin{array}{l}0 \\
0\end{array}$ & $\begin{array}{l}3 \\
0\end{array}$ & $\begin{array}{l}5 \\
0\end{array}$ & $\begin{array}{l}0 \\
0\end{array}$ \\
\hline 1970 & $\frac{\mathrm{M}}{\mathrm{F}}$ & $\begin{array}{l}59 \\
43\end{array}$ & $\begin{array}{l}2 \\
2\end{array}$ & $\begin{array}{l}0 \\
0\end{array}$ & $\begin{array}{l}2 \\
0\end{array}$ & $\begin{array}{l}0 \\
0\end{array}$ & $\begin{array}{l}5 \\
0\end{array}$ & $\begin{array}{l}0 \\
0\end{array}$ & $\begin{array}{l}5 \\
0\end{array}$ & $\begin{array}{l}4 \\
0\end{array}$ & $\begin{array}{l}0 \\
0\end{array}$ \\
\hline 1971 & $\frac{\mathrm{M}}{\mathrm{F}}$ & $\begin{array}{l}76 \\
62\end{array}$ & $\begin{array}{l}1 \\
1\end{array}$ & $\begin{array}{l}0 \\
0\end{array}$ & $\begin{array}{l}4 \\
3\end{array}$ & $\begin{array}{l}0 \\
0\end{array}$ & $\begin{array}{l}3 \\
0\end{array}$ & $\begin{array}{l}0 \\
0\end{array}$ & $\begin{array}{l}6 \\
1\end{array}$ & $\begin{array}{l}2 \\
1\end{array}$ & $\begin{array}{l}0 \\
0\end{array}$ \\
\hline 1972 & $\frac{\mathrm{M}}{\mathrm{F}}$ & $\begin{array}{l}81 \\
61\end{array}$ & $\begin{array}{l}1 \\
0\end{array}$ & $\begin{array}{l}0 \\
0\end{array}$ & $\begin{array}{l}1 \\
3\end{array}$ & $\begin{array}{l}3 \\
0\end{array}$ & $\begin{array}{l}0 \\
0\end{array}$ & $\begin{array}{l}0 \\
1\end{array}$ & $\begin{array}{l}4 \\
1\end{array}$ & $\begin{array}{l}5 \\
2\end{array}$ & $\begin{array}{l}0 \\
0\end{array}$ \\
\hline 1973 & $\begin{array}{l}\mathrm{M} \\
\mathrm{F}\end{array}$ & $\begin{array}{l}151 \\
109 \\
\end{array}$ & $\begin{array}{l}4 \\
2\end{array}$ & $\begin{array}{l}0 \\
0\end{array}$ & $\begin{array}{r}11 \\
5\end{array}$ & $\begin{array}{l}1 \\
0\end{array}$ & $\begin{array}{l}1 \\
0\end{array}$ & $\begin{array}{l}0 \\
0\end{array}$ & $\begin{array}{l}0 \\
0\end{array}$ & $\begin{array}{l}1 \\
1\end{array}$ & $\begin{array}{l}0 \\
0\end{array}$ \\
\hline Total & $\frac{\mathrm{M}}{\mathrm{F}}$ & $\begin{array}{l}948 \\
619\end{array}$ & $\begin{array}{l}17 \\
15\end{array}$ & $\begin{array}{l}0 \\
0\end{array}$ & $\begin{array}{l}60 \\
26\end{array}$ & $\begin{array}{l}4 \\
1\end{array}$ & $\begin{array}{r}16 \\
5\end{array}$ & $\begin{array}{l}0 \\
1\end{array}$ & $\begin{array}{r}44 \\
6\end{array}$ & $\begin{array}{l}51 \\
12\end{array}$ & $\begin{array}{l}3 \\
0\end{array}$ \\
\hline
\end{tabular}


TABLE 10

Fibrosis and cirrhosis not associated with hepatocellular nor cholangiocarcinoma among autopsies in Japan (1958 - 1973)

\begin{tabular}{|c|c|c|c|c|c|c|c|c|c|c|c|}
\hline Year & Sex & Totl & Fibr & Fat $\mathrm{C}$ & Bil C & Card & A typ & $\mathrm{A}^{\prime}$ typ & B typ & C NOS & $B^{\prime}$ typ \\
\hline 1958 & $\frac{\mathrm{M}}{\mathrm{F}}$ & $\begin{array}{l}241 \\
140\end{array}$ & $\begin{array}{l}21 \\
11\end{array}$ & $\begin{array}{l}2 \\
1\end{array}$ & $\begin{array}{l}31 \\
32\end{array}$ & $\begin{array}{l}18 \\
11\end{array}$ & $\begin{array}{l}25 \\
15\end{array}$ & $\begin{array}{l}0 \\
0\end{array}$ & $\begin{array}{l}72 \\
30\end{array}$ & $\begin{array}{l}70 \\
39\end{array}$ & $\begin{array}{l}2 \\
7\end{array}$ \\
\hline 1959 & $\frac{\mathrm{M}}{\mathrm{F}}$ & $\begin{array}{l}312 \\
152\end{array}$ & $\begin{array}{l}33 \\
19\end{array}$ & $\begin{array}{l}2 \\
2\end{array}$ & $\begin{array}{l}56 \\
24\end{array}$ & $\begin{array}{l}26 \\
12\end{array}$ & $\begin{array}{l}28 \\
19\end{array}$ & $\begin{array}{l}0 \\
0\end{array}$ & $\begin{array}{l}78 \\
36\end{array}$ & $\begin{array}{l}82 \\
37\end{array}$ & $\begin{array}{l}7 \\
3\end{array}$ \\
\hline 1960 & $\frac{\mathrm{M}}{\mathrm{F}}$ & $\begin{array}{l}340 \\
143\end{array}$ & $\begin{array}{l}28 \\
20\end{array}$ & $\begin{array}{l}2 \\
0\end{array}$ & $\begin{array}{l}43 \\
25\end{array}$ & $\begin{array}{l}25 \\
11\end{array}$ & $\begin{array}{l}46 \\
23\end{array}$ & $\begin{array}{l}0 \\
0\end{array}$ & $\begin{array}{l}96 \\
26\end{array}$ & $\begin{array}{l}93 \\
35\end{array}$ & $\begin{array}{l}7 \\
3\end{array}$ \\
\hline 1961 & $\frac{\mathrm{M}}{\mathrm{F}}$ & $\begin{array}{l}417 \\
194\end{array}$ & $\begin{array}{l}46 \\
25\end{array}$ & $\begin{array}{l}5 \\
0\end{array}$ & $\begin{array}{l}55 \\
43\end{array}$ & $\begin{array}{l}24 \\
12\end{array}$ & $\begin{array}{l}52 \\
23\end{array}$ & $\begin{array}{l}1 \\
0\end{array}$ & $\begin{array}{r}109 \\
38\end{array}$ & $\begin{array}{r}121 \\
51\end{array}$ & $\begin{array}{l}4 \\
2\end{array}$ \\
\hline 1962 & $\frac{\mathrm{M}}{\mathrm{F}}$ & $\begin{array}{l}481 \\
221\end{array}$ & $\begin{array}{l}51 \\
34\end{array}$ & $\begin{array}{l}8 \\
0\end{array}$ & $\begin{array}{l}67 \\
36\end{array}$ & $\begin{array}{l}32 \\
18\end{array}$ & $\begin{array}{l}53 \\
28\end{array}$ & $\begin{array}{l}5 \\
2\end{array}$ & $\begin{array}{r}107 \\
44\end{array}$ & $\begin{array}{r}145 \\
53\end{array}$ & $\begin{array}{r}13 \\
6\end{array}$ \\
\hline 1963 & $\frac{\mathrm{M}}{\mathrm{F}}$ & $\begin{array}{l}502 \\
203\end{array}$ & $\begin{array}{l}73 \\
28\end{array}$ & $\begin{array}{l}9 \\
0\end{array}$ & $\begin{array}{l}62 \\
33\end{array}$ & $\begin{array}{l}26 \\
18\end{array}$ & $\begin{array}{l}64 \\
29\end{array}$ & $\begin{array}{l}1 \\
0\end{array}$ & $\begin{array}{r}111 \\
33\end{array}$ & $\begin{array}{r}141 \\
57\end{array}$ & $\begin{array}{r}15 \\
5\end{array}$ \\
\hline 1964 & $\frac{\mathrm{M}}{\mathrm{F}}$ & $\begin{array}{l}528 \\
266\end{array}$ & $\begin{array}{l}67 \\
36\end{array}$ & $\begin{array}{l}4 \\
3\end{array}$ & $\begin{array}{l}63 \\
59\end{array}$ & $\begin{array}{l}28 \\
13\end{array}$ & $\begin{array}{l}78 \\
42\end{array}$ & $\begin{array}{l}6 \\
2\end{array}$ & $\begin{array}{l}99 \\
42\end{array}$ & $\begin{array}{r}169 \\
66\end{array}$ & $\begin{array}{r}14 \\
3\end{array}$ \\
\hline 1965 & $\frac{\mathrm{M}}{\mathrm{F}}$ & $\begin{array}{l}621 \\
258\end{array}$ & $\begin{array}{l}90 \\
30\end{array}$ & $\begin{array}{l}8 \\
5\end{array}$ & $\begin{array}{l}71 \\
51\end{array}$ & $\begin{array}{l}25 \\
15\end{array}$ & $\begin{array}{l}81 \\
38\end{array}$ & $\begin{array}{l}2 \\
0\end{array}$ & $\begin{array}{r}123 \\
49\end{array}$ & $\begin{array}{r}209 \\
68\end{array}$ & $\begin{array}{r}12 \\
2\end{array}$ \\
\hline 1966 & $\frac{M}{F}$ & $\begin{array}{l}701 \\
299\end{array}$ & $\begin{array}{r}118 \\
63\end{array}$ & $\begin{array}{l}3 \\
3\end{array}$ & $\begin{array}{l}67 \\
42\end{array}$ & $\begin{array}{l}21 \\
16\end{array}$ & $\begin{array}{r}113 \\
38\end{array}$ & $\begin{array}{l}5 \\
5\end{array}$ & $\begin{array}{r}142 \\
43\end{array}$ & $\begin{array}{r}219 \\
82\end{array}$ & $\begin{array}{r}13 \\
7\end{array}$ \\
\hline 1967 & $\frac{\mathrm{M}}{\mathrm{F}}$ & $\begin{array}{l}760 \\
331\end{array}$ & $\begin{array}{r}140 \\
66\end{array}$ & $\begin{array}{r}13 \\
7\end{array}$ & $\begin{array}{l}60 \\
57\end{array}$ & $\begin{array}{l}27 \\
22\end{array}$ & $\begin{array}{l}76 \\
35\end{array}$ & $\begin{array}{r}17 \\
6\end{array}$ & $\begin{array}{r}197 \\
62\end{array}$ & $\begin{array}{r}217 \\
74\end{array}$ & $\begin{array}{r}13 \\
2\end{array}$ \\
\hline 1968 & $\frac{\mathrm{M}}{\mathrm{F}}$ & $\begin{array}{l}959 \\
453\end{array}$ & $\begin{array}{r}152 \\
72\end{array}$ & $\begin{array}{r}20 \\
6\end{array}$ & $\begin{array}{l}78 \\
58\end{array}$ & $\begin{array}{l}38 \\
23\end{array}$ & $\begin{array}{r}134 \\
63\end{array}$ & $\begin{array}{l}26 \\
13\end{array}$ & $\begin{array}{r}216 \\
74\end{array}$ & $\begin{array}{l}282 \\
136\end{array}$ & $\begin{array}{r}13 \\
8\end{array}$ \\
\hline 1969 & $\frac{\mathrm{M}}{\mathrm{F}}$ & $\begin{array}{l}929 \\
495\end{array}$ & $\begin{array}{r}141 \\
88\end{array}$ & $\begin{array}{r}22 \\
8\end{array}$ & $\begin{array}{l}66 \\
66\end{array}$ & $\begin{array}{l}35 \\
25\end{array}$ & $\begin{array}{r}136 \\
57\end{array}$ & $\begin{array}{l}7 \\
8\end{array}$ & $\begin{array}{l}238 \\
106\end{array}$ & $\begin{array}{l}266 \\
134\end{array}$ & $\begin{array}{r}18 \\
3\end{array}$ \\
\hline 1970 & $\frac{\mathrm{M}}{\mathrm{F}}$ & $\begin{array}{l}847 \\
428\end{array}$ & $\begin{array}{r}132 \\
76\end{array}$ & $\begin{array}{r}13 \\
3\end{array}$ & $\begin{array}{l}60 \\
51\end{array}$ & $\begin{array}{l}31 \\
20\end{array}$ & $\begin{array}{r}158 \\
70\end{array}$ & $\begin{array}{l}3 \\
2\end{array}$ & $\begin{array}{l}250 \\
107\end{array}$ & $\begin{array}{r}196 \\
99\end{array}$ & $\begin{array}{l}4 \\
0\end{array}$ \\
\hline 1971 & $\frac{\mathrm{M}}{\mathrm{F}}$ & $\begin{array}{l}895 \\
415\end{array}$ & $\begin{array}{r}137 \\
87\end{array}$ & $\begin{array}{r}19 \\
8\end{array}$ & $\begin{array}{l}54 \\
50\end{array}$ & $\begin{array}{l}30 \\
27\end{array}$ & $\begin{array}{r}136 \\
65\end{array}$ & $\begin{array}{r}26 \\
8\end{array}$ & $\begin{array}{r}306 \\
91\end{array}$ & $\begin{array}{r}160 \\
70\end{array}$ & $\begin{array}{r}27 \\
9\end{array}$ \\
\hline 1972 & $\frac{\mathrm{M}}{\mathrm{F}}$ & $\begin{array}{l}845 \\
403\end{array}$ & $\begin{array}{r}119 \\
66\end{array}$ & $\begin{array}{r}24 \\
3\end{array}$ & $\begin{array}{l}68 \\
53\end{array}$ & $\begin{array}{l}42 \\
13\end{array}$ & $\begin{array}{r}131 \\
67\end{array}$ & $\begin{array}{l}36 \\
13\end{array}$ & $\begin{array}{l}268 \\
121\end{array}$ & $\begin{array}{r}136 \\
55\end{array}$ & $\begin{array}{l}21 \\
12\end{array}$ \\
\hline 1973 & $\frac{\mathrm{M}}{\mathrm{F}}$ & $\begin{array}{r}1,017 \\
340\end{array}$ & $\begin{array}{r}141 \\
58\end{array}$ & $\begin{array}{r}41 \\
6\end{array}$ & $\begin{array}{l}78 \\
26\end{array}$ & $\begin{array}{l}23 \\
18\end{array}$ & $\begin{array}{r}151 \\
49 \\
\end{array}$ & $\begin{array}{l}57 \\
13\end{array}$ & $\begin{array}{l}346 \\
118\end{array}$ & $\begin{array}{r}149 \\
45\end{array}$ & $\begin{array}{r}31 \\
7\end{array}$ \\
\hline Total & $\frac{\mathrm{M}}{\mathrm{F}}$ & $\begin{array}{r}10,395 \\
4,741\end{array}$ & $\begin{array}{r}1,489 \\
779\end{array}$ & $\begin{array}{r}195 \\
55\end{array}$ & $\begin{array}{l}979 \\
706\end{array}$ & $\begin{array}{l}451 \\
274\end{array}$ & $\begin{array}{r}1,462 \\
661\end{array}$ & $\begin{array}{r}192 \\
72\end{array}$ & $\begin{array}{l}2,758 \\
1,020\end{array}$ & \begin{tabular}{|l|}
2.655 \\
1.101
\end{tabular} & $\begin{array}{r}214 \\
73\end{array}$ \\
\hline
\end{tabular}

TABLE 11

Cirrhotic changes associated and not associated with malignacies(\%)

\begin{tabular}{|c|c|c|c|c|c|c|c|c|c|c|c|}
\hline Male & Total & $\begin{array}{l}\text { With } \\
\text { cirr }\end{array}$ & Fibr & Fatt & Bili & Card & A & $A^{\prime}$ & B & $\begin{array}{l}\text { Cirr } \\
\text { NOS }\end{array}$ & $\mathrm{B}^{\prime}$ \\
\hline None & 10,395 & & 14.3 & 1.9 & 9.4 & 4.3 & 14.1 & 1.8 & 26.5 & 25.5 & 2.1 \\
\hline Hepa. & 5,660 & 3,961 & 1.6 & 0.1 & 0.5 & 0.2 & 8.2 & 2.0 & 44.1 & 41.7 & 1.6 \\
\hline Chol. & 948 & 196 & 8.7 & 0 & 30.6 & 2.0 & 8.2 & 0 & 22.4 & 26.0 & 2.0 \\
\hline \multicolumn{12}{|c|}{ Female } \\
\hline None & 4,741 & & 16.4 & 1.2 & 14.9 & 5.8 & 13.9 & 1.5 & 21.5 & 23.2 & 1.5 \\
\hline Нера. & 1,331 & 709 & 2.0 & 0 & 1.0 & 0.6 & 10.2 & 2.7 & 43.0 & 37.8 & 2.8 \\
\hline Chol. & 619 & 66 & 22.0 & 0 & 39.4 & 1.5 & 7.6 & 1.5 & 9.1 & 18.2 & 0 \\
\hline
\end{tabular}


TABLE 12

Association of hepatocellular carcinoma with cirrhotic changes among 275, 857 autopsies during 16 years from 1958 to 1973

\begin{tabular}{c|cc|c}
\hline & Male & Female & Total \\
\hline $\begin{array}{l}\text { Hepatocellular Ca } \\
\text { with cirrhot. changes } \\
(\%)\end{array}$ & 70.0 & 53.3 & 66.8 \\
$\begin{array}{c}\text { Cirrhot. changes with } \\
\text { hepatocellular Ca } \\
(\%)\end{array}$ & 27.2 & 12.9 & 23.3 \\
\hline
\end{tabular}

of intrahepatic cholangiocarcinoma (cholangiocellular carcinoma) with cirrhotic changes is shown in Tables 11 and 13. It shows that 20.7 percent of cholangiocellular carcinoma in male and 10.7 percent in female are associated with cirrhotic changes of the liver.

The association of fibrosis of the liver with hepatecellular carcinoma in male and female was found as 1.6 percent and 2.0 percent respectively, as shown in Table 11, which did not show any statistically significant difference of sex. Cholangiocellular carcinoma is also associated with fibrosis of the liver in the ratio of 8.7 percent in male and 22.0 percent in female with statistically significant difference of sex $(P>$ $0.05)$.

As shown in Table 8, there has been an increasing tendency of A and A' type cirrhosis associated with hepatocellular carcinoma from 1958 to 1973. Those cirrhoses not associated with hepatocellular carcinoma have also increased in number during the same period of time as shown in Table 10. There was a close association of biliary cirrhosis with cholangiocellular carcinoma compared with hepatocellular carcinoma, due possibly to secondary biliary cirrhosis caused by obstruction of bile duct with neoplasm.
TABLE 13

Association of cholangiocellular carcinoma with cirrhotic changes among 275, 857 autopsies during 16 years from 1958 to 1973

\begin{tabular}{c|cc|c}
\hline & Male & Female & Total \\
\hline $\begin{array}{c}\text { Cholangiocellular Ca } \\
\text { with cirrhotic change } \\
(\%)\end{array}$ & 20.7 & 10.7 & 16.7 \\
$\begin{array}{c}\text { Cirrhotic changes with } \\
\text { cholangiocellular Ca } \\
(\%)\end{array}$ & 1.7 & 1.4 & 1.7 \\
\hline
\end{tabular}

Table 11 shows that the majority of cirrhoses associated with hepatocellular carcinoma were B type cirrhosis and cirrhosis NOS (Not Otherwise Specified), which amounted to 85.8 per cent in male and 80.8 percent in female. Among cirrhoses not associated with hepatocellular and cholangiocellular carcinoma, as shown in Table 10 and 11 , $B$ type cirrhosis and cirrhosis NOS were found in the ratio of 50.0 percent in male and 44.7 per cent in female, revealing that these lesions are frequent cirrhoses irrespective of the fact that whether these were associated with hepatocellular carcinoma or not.

On the other hand, B type cirrhosis and cirrhosis NOS are closely associated with hepatocellular carcinoma in the ratio of 38.3 and 37.9 percent in male, and 22.9 and 19.4 percent in female respectively. As far as percentages are concerned, a close association of $\mathrm{A}^{\prime}$ and B' type cirrhosis with hepatocellular carcinoma is also noted. The ratio is 29.7 and 23.2 percent in male and 20.7 and 21.5 percent in female. However, the number of these cirrhotic changes is much smaller compared with B type and NOS cirrhosis.

Cholangiocellular carcinoma, in contrast to hepatocellular carcinoma, showed a less association with cirrhosis as 
shown in Table 13. It was also noted from Tables 2, 8, 9 and 10 that the frequency of $B$ type cirrhosis among total autopsies is 2.77 percent in male and 1.21 percent in female, and that of cirrhosis NOS 2.65 percent in male and 1.21 per cent in female. From these figures, it is assumed that cholangiocellular carcinoma has not much to do with any type of cirrhosis except biliary cirrhosis, and cirrhosis itself has almost nothing to do with cholangiocellular carcinoma.

4. Age-specific incidence of hexatocellular carcinoma and cirrhotic changes

Fig. 2 to 4 illustrate age incidence of hepatocellular carcinoma, B type cirrhosis and cirrhosis NOS in male and female. The peaks of hepatocellular carcinoma in male and female are at

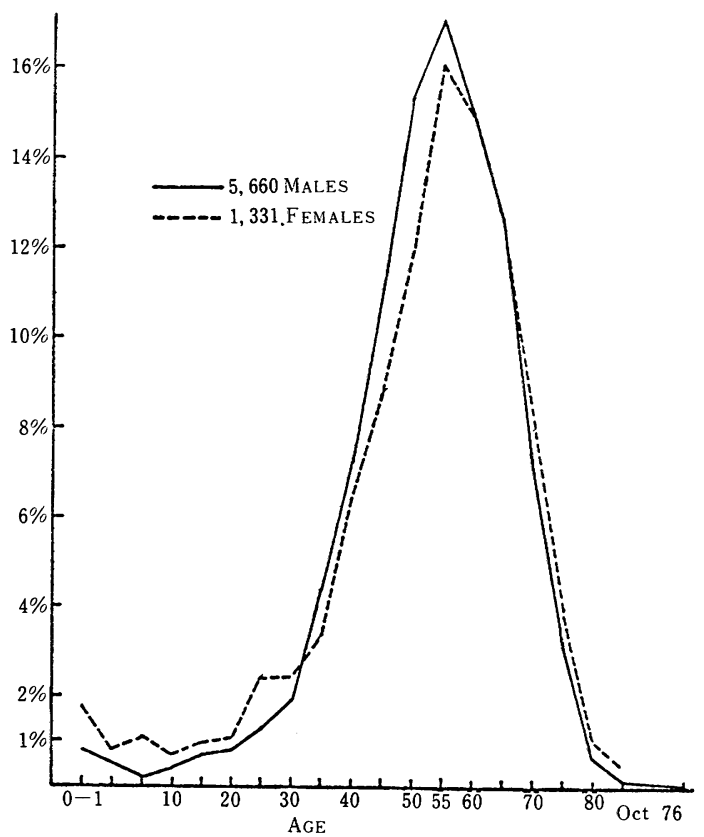

Fig. 2 Age distribution of autopsied patients with hepatocellular carcinoma (1958-1973).

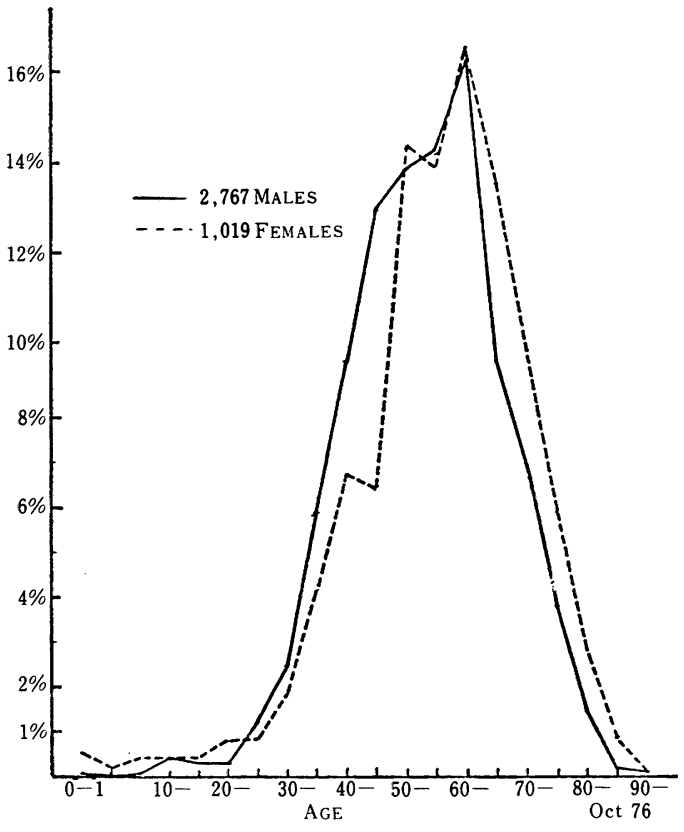

Fig. 3 Age distribution of autopsied patients with B type cirrhosis (posthepatitic) $(1958-1973)$.

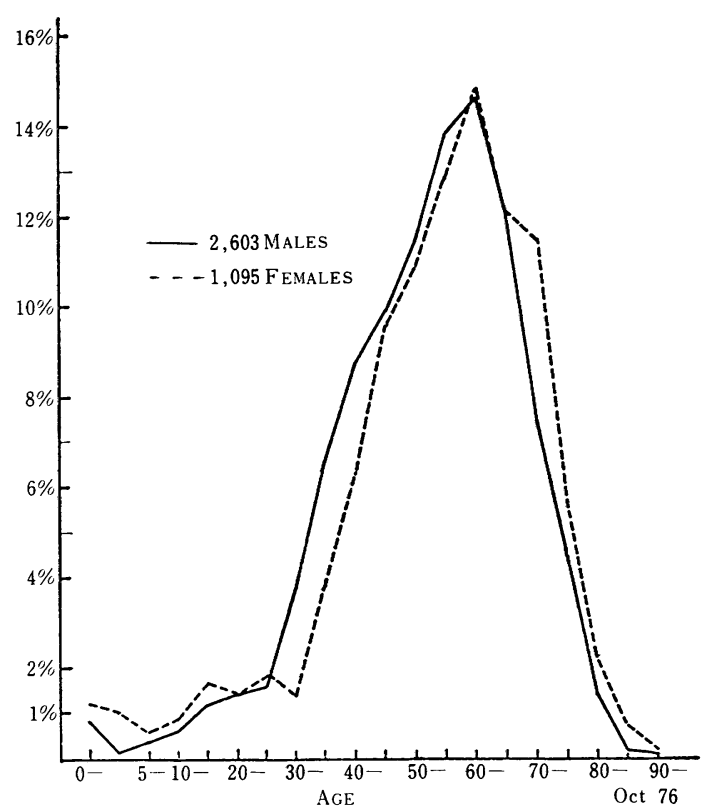

Fig. 4 Age distribution of autopsied patients with cirrhosis NOS (1958-1973). 
the age of 55 , and those of $B$ type and NOS cirrhosis are at the age of 60 . It was not postulated whether the cirrhotic changes are precursor of hepatocellular carcinoma or not, but slight abnormality of age incidence of B type cirrhosis was noted in male as well as in female. A small crest of cirrhosis NOS at the age group of $0-2$ led to the belief that some of them could be attributed to congenital anomalies of the bile duct, as biliary cirrhosis reaches a peak at the age group of $0-2$, which was caused mostly by congenital atresia or obstruction of intra- and extrahepatic bile ducts.

As Fig. 2 shows that comparatively few cases are in the age group younger than 25 years, those cases are deducted in Table 14, which shows hepatocellular carcinoma of older than 25 years of age, to which 96.2 percent of cases (96. 8 percent in male and 93.5 percent in female) belonged.

\section{DISCUSSION}

Increasing incidence of cirrhosis among autopsy cases has been noted by many authors (Caroli et al., 1970; Knopp, 1967 ; MacDonald, 1956 ; Manderson et al., 1965 ; Purtilo and Gottlieb, 1973 ; Schmitz, 1971). MacDonald (MacDonald, 1956) has observed an increase of hepatocellular carcinoma among autopsy cases with the increase of cirrhosis in number, and the same effect has been demonstrated by Péquignot (Péquignot et al., 1967), Epstein (Epstein, 1964), Manderson (Manderson et al., 1965), and Elkington (Elkington et al., 1963). However, the incidences of hepatocellular carcinoma in their autopsy cases have been much smaller than those of Japan, 3.26 percent in male and 1.13 percent in female as shown in Table 15. Most of these figures were
TABLE 14

Frequency of hepatocellular carcinoma among autopsies of over 25-years-old during 16 years from 1958 to 1973

\begin{tabular}{|c|c|c|c|c|}
\hline \multirow{2}{*}{ Year } & \multicolumn{2}{|c|}{ Total } & \multicolumn{2}{|c|}{ Hepat. Ca. } \\
\hline & $\mathrm{M}$ & F & $\mathrm{M}$ & $\mathrm{F}$ \\
\hline 1958 & 3,763 & 2,201 & 114 & 28 \\
\hline 1959 & 3,749 & 2,451 & 115 & 30 \\
\hline 1960 & 4,316 & 2,573 & 138 & 40 \\
\hline 1961 & 4,834 & 3,166 & 164 & 46 \\
\hline 1962 & 5,293 & 3,535 & 190 & 28 \\
\hline 1963 & 6,209 & 3,904 & 210 & 43 \\
\hline 1964 & 6,697 & 4,098 & 253 & 67 \\
\hline 1965 & 7,662 & 4,602 & 283 & 56 \\
\hline 1966 & 8,138 & 5,274 & 337 & 77 \\
\hline 1967 & 9,589 & 6,163 & 373 & 94 \\
\hline 1968 & 11,824 & 7,737 & 531 & 105 \\
\hline 1969 & 11,165 & 7,362 & 492 & 108 \\
\hline 1970 & 10,322 & 6,788 & 531 & 105 \\
\hline 1971 & 10,319 & 6,627 & 573 & 128 \\
\hline 1972 & 10,723 & 6,959 & 625 & 134 \\
\hline 1973 & 11,118 & 7,253 & 551 & 156 \\
\hline Total & 125,72 & 180,69 & 5,480 & 1,245 \\
\hline
\end{tabular}

less than 1 percent except for those from Africa (G. Thomson), India (Scrinivasa and Sharman, 1968) and China (Ying et al., 1963). On the other hand, the incidence of cirrhosis among autopsy cases in Japan, taking cirrhosis of unknown etiology (A, A', B, B' type and NOS), was 5.44 percent (6.87 per cent in male and 3.32 in female) and 3.73 percent (4. 43 percent in male and 2.67 percent in female) excluding those associated with hepatocellular and cholangiocelluar carcinoma, as calculated from Tables 8, 9 and 10. These figures were not large comared with those of other countries, as shown in Table 16. Hence, it is noted that the incidence of hepatocellular carcinoma is considerably high among autopsy cases and in the Vital Statistics in 
spite of the fact that the incidence of cirrhosis is not high compared with other countries. However, the cirrhosis in Japan, mostly B type and NOS, is extraordinarily frequently associated with hepatocellular carcinoma, thus elevating the incidence of hepatocelluIar carcinoma to certain extent.

\section{TABLE 15}

Frequency of hepatocellular carcinoma and cholangiocarcinoma among autopsy cases in the world

\begin{tabular}{|c|c|c|c|c|c|}
\hline Author (s) & Country & $\begin{array}{c}\text { Number of } \\
\text { autopsies }\end{array}$ & $\begin{array}{c}\text { Number } \\
\text { of } \\
\text { H.C. }\end{array}$ & $\begin{array}{c}\% \\
\text { of } \\
\text { H.C. }\end{array}$ & $\begin{array}{l}\text { Number } \\
\text { of } \\
\text { cholan- } \\
\text { gioca. }\end{array}$ \\
\hline Edmondson and Steiner (1954) & U.S.A. & 48,900 & 75 & 0.15 & 18 \\
\hline Köhn, K. (1955) & Germany & 35,745 & 80 & 0.22 & - \\
\hline Roulet, F. C. (1977) & Switzerland & 23,470 & 93 & 0.40 & 16 \\
\hline MacDonald, R. A. (1956) & U.S.A. & 23,114 & 80 & - & 24 \\
\hline Berman, C. (1958) & South Africa & 15,280 & 248 & 1.62 & - \\
\hline Gall, E. A. (1960) & U.S.A. & 12,200 (over $21 \mathrm{y}$. ) & 76 & 0.62 & 20 \\
\hline Becker and Chatgidakis (1961) & South Africa & 4,949 (white) & 9 & 0.18 & 0 \\
\hline " & " & 4,893 (native) & 84 & 1.72 & 6 \\
\hline Wainwright, J. (1961) & South Africa & 2,560 & 102 & 3.98 & 14 \\
\hline Shanmugaratnam, K. (1961) & Singapore & 9,893 & 103 & - & 9 \\
\hline Glennert, J. (1961) & Denmark & 14,881 & 50 & 0.34 & 36 \\
\hline Tiktiniski, O. L. (1961) & U.S.S.R. & 6,163 & 17 & 0.28 & - \\
\hline Ying et al. (1963) & China & 3,497 & 87 & 2.49 & 10 \\
\hline Elkington et al. (1963) & England & 7,366 & 39 & 0.53 & - \\
\hline Sagebiel et al. (1963) & U.S.A. & 23,275 & 100 & 0.43 & - \\
\hline Sundarsanam et al. (1963) & India & 3,400 & 22 & 0.65 & 2 \\
\hline Miyai and Reubner (1963) & U.S.A. & 25,000 & 64 & 0.26 & 8 \\
\hline Ervasti, J. (1964) & Finland & 15,545 & 45 & 0.29 & 29 \\
\hline Patton and Horn (1964) & U.S.A. & 12,980 & 47 & 0.36 & 13 \\
\hline Epstein, S. (1964) & U.S.A. & 3,079 & 50 & 1.62 & - \\
\hline Saragoca et al. (1964) & Portugal & 168 & 6 & 3.57 & - \\
\hline " & " & 4,338 & 14 & 0.32 & - \\
\hline Ohlsson and Nordén (1965) & Sweden & 8,837 & 120 & 1.36 & 1 \\
\hline San Jose et al. (1965) & U.S.A. & 12,687 & 73 & 0.58 & 6 \\
\hline Manderson et al. (1965) & England & 6,586 & 41 & 0.62 & - \\
\hline Ottolenghi, A. (1966) & Italy & 6,241 & 94 & 1.51 & - \\
\hline " & " & 4,000 & 124 & 3.10 & - \\
\hline Péquignot et al. (1967) & France & 2,540 & 42 & 1.65 & - \\
\hline Voigt and Helbig (1968) & Germany & 26.235 & 75 & 0.29 & 42 \\
\hline Scrinivasa and Sharma (1938) & India & 1,293 & 29 & 2.24 & 2 \\
\hline Wöckel and Altrock (1968) & Germany & 15,568 & 40 & 0.26 & - \\
\hline Sievers, B. -U. (1973) & Germany & 4,411 & 36 & 0.82 & 11 \\
\hline Purtilo and Gottlieb (1973) & U.S.A. & 14,000 & 84 & 0.60 & 12 \\
\hline Elsner and Jauregui (1974) & Argentina & 22,170 & 54 & 0.24 & 10 \\
\hline
\end{tabular}

H. C., hepatocellular carcinoma. 
With the progress of treatment of cirrhois, cirrhotic patients can expect a longer survival than before, which has been attributed to an increasing incidence of hepatocellular carcinoma (Caroli et al., 1970; MacDonald, 1956 ; Péquignot et al., 1967). However, as mentioned above, the incidence of cirrhosis among autopsy cases is not high compared with Western countries, yet cirrhotic changes in Japan has shown a close association with hepatocellular carcinoma. It is considered that the backround of Japanese people might be

TABLE 16

Frequency of cirrhosis among autopsy cases in the world

\begin{tabular}{|c|c|c|c|c|}
\hline Author (s) & Country & $\begin{array}{l}\text { Number of } \\
\text { autopsies }\end{array}$ & $\begin{array}{c}\text { Number } \\
\text { of } \\
\text { cirrhosis }^{a}\end{array}$ & $\begin{array}{c}\% \text { of } \\
\text { cirrhosis }\end{array}$ \\
\hline Hall and Sun (1951) & U.S.A. & 12,000 & 490 & 4.08 \\
\hline Meyer, P. G. (1951) & Germany & 20,295 & 575 & 2.83 \\
\hline Lunzenauer, K. (1955) & Germany & 26,515 & 603 & 2.27 \\
\hline Parker, R. G. F. (1957) & England & 17,732 & 184 & 1.04 \\
\hline Dible, J. H. (1957) & England & 7,217 & 109 & 1.51 \\
\hline Gall, E. A. (1960) & U.S.A. & 16,332 & 654 & - \\
\hline Riva and Weiner (1960) & Switzerland & 5,482 & 331 & 6.04 \\
\hline Börner, F. (1961) & Germany & 3,129 & 142 & 4.54 \\
\hline \multirow[t]{3}{*}{ Thomson, G. (1961) } & \multirow[t]{3}{*}{ South Africa } & 1,473 (white) & 77 & 5.23 \\
\hline & & 1, 319 (colored) & 22 & 1.67 \\
\hline & & 358 (aborigine) & 22 & 6.15 \\
\hline \multirow[t]{2}{*}{ Becker and Chatgidakis (1961) } & \multirow[t]{2}{*}{ South Africa } & 4,949 (white) & 291 & 5.88 \\
\hline & & 4,893 (aborigine) & 242 & 4.95 \\
\hline \multirow[t]{2}{*}{ Wainwright, J. (1961) } & \multirow[t]{2}{*}{ South Africa } & 1,326 (white) & 95 & 7.16 \\
\hline & & 2,560 (aborigine) & 183 & 7.15 \\
\hline Shanmugaratnam, K. (1961) & Singapore & 9,893 & 353 & 3.57 \\
\hline \multirow[t]{2}{*}{ Knopp, J. (1961) } & \multirow[t]{2}{*}{ Germany } & 55,587 & 1,628 & 2.93 \\
\hline & & 5,175 & 555 & 10.72 \\
\hline MacDonald, R. A. (1956) & U.S.A. & 23,114 & 1,824 & 7.89 \\
\hline Tung et al. (1964) & China & 6,468 & - & 9.49 \\
\hline Elkington et al. (1963) & England & 7,366 & 157 & 2.13 \\
\hline Miyai and Ruebner (1963) & U.S.A. & 25,000 & 446 & 1.86 \\
\hline Borel, C. A. (1964) & Switzerland & 10,000 & 787 & 7.87 \\
\hline Ervasti, J.. (1964) & Finland & 15,545 & 478 & 3.07 \\
\hline Ohlsson and Norden (1965) & Sweden & 8,837 & 271 & 3.07 \\
\hline Ottolenghi, A. (1966) & Italy & 6,241 & 649 & 10.40 \\
\hline Péquignot, H. et al. (1967) & France & 2,540 & 373 & 14.69 \\
\hline Voigt and Helbig (1968) & Germany & 26,235 & 1,166 & 4.44 \\
\hline Wöckel and Altrock (1968) & Germany & 15,568 & 458 & 2.94 \\
\hline Lopez-Corella et al. (1968) & Mexico & 6,558 & 880 & 13.42 \\
\hline Purtilo and Gottlieb (1973) & U.S.A. & 14,000 & - & 11.5 \\
\hline Elsner and Jauregui (1974) & Argentina & 22,170 & 805 & 3.63 \\
\hline
\end{tabular}

${ }^{\text {a }}$ Cirrhoses of known etiology such as biliary and cardiac were excluded as much as possible. 
different from Western people in respect to viral infection, nutritional status, environmental factors, ethnic and demographic characteristics.

Alcoholic beverages have been implicated in cirrhosis as a potential cause. Recently, Purtilo and Gottlieb (Purtilo and Gottlieb, 1973) have reported progressively increased frequency of cirrhosis and hepatocellular carcinoma, especially cirrhosis associated with chronic alcoholism in Boston City Hosptial. They noted that alcoholic cirrhosis or fatty nutritional cirrhosis increased from 31 percent (1971-1926) to 61 percent $(1955-1968)$. Sievers (Sievers, 1973) also reported that as an etiological cause of cirrhosis, chronic alcoholism was found to the extent of 46.8 percent. Leevy et al. (Leevy et al., 1964) demonstrated by multiple biopsies that alcoholic cirrhosis sometimes progressed to postnecrotic cirrhosis, hemochromatosis and hepatoma. Sakurai (Sakurai, 1969), who has studied the influence of alcohol in the development cirrhosis on 4,032 consecutive autopsies in Japan, reported that there was remarkable influence of Japanese rice wine on the production of cirrhosis, as there was marked difference between drinkers and nondrinker among patients with cirrhosis and hepatoma. However, he also added that Japanese rice wine is mildly toxic to the liver, and it was exceptionally rare to see typical histologic features of alcoholic liver disease in Japan.

From the peculiar geographical distribution of hepatocellular carcinoma in the world, nutritional deficiency has been considered as an important cause of the lesion (Berman, 1951). It is possible that undernutrition might make the liver liable to be injured by noxious agents (Kasliwa and Gupta, 1963), particularly in view of the fact that incidences of hepatocelluiar carcinoma of colored and Asian people lowered sharply when they immigrated to the United States. Koizumi and others (Koizumi et al., 1971) studied dietary habits of 30 patients with hepatocellular carcinoma and found that there was a difference concerning calory, protein and vegetables compared with the controls when they were young, and had a tendency of less intake of protein all thrhough their lives.

Viral hepatitis undoubtedly has close association with cirrhosis, and it has been demonstrated by epidemiological studies and by use of multiple biopsies that viral hepatitis transformed into cirrhosis and sometimes developed hepatocellular carcinoma in the long run (Kosaka, 1963 ; Nielsen, 1971; Prince, 1970 ; Purtilo and Gottlieb, 1973). Since the discovery of hepatitis $B$ antigen (HBAg) by G. S. Blumberg in 1965, association of viral hepatitis with cirrhosis became more evident (Prince, 1970; Nishioka, 1972), and familial clustering of HBAg has been demonstrated (Ohbayashi et al., 1972; Ohbayashi and Mayumi, 1974). HBAg has been positive in 34.1 percent of cirrhosis and in 48.3 percent of hepatocellular! carcinoma (Nishioka, 1972). However, very few patients suffering from viral hepatitis develop cirrhosis and hepatocellular carcinoma, and the reason for this exception remains to be studied.

Aflatoxin has been found as a potent carcinogen to the liver, and Alpert (Alpert et al., 1969) found 29.6 percent of 480 food samples collected in Uganda contained aflatoxin at detectable levels, and 3.7 percent of them contained more than $1 \mathrm{ng} / \mathrm{kg}$. He also added that in the area of high incidence of hepatocellular carcinoma the frequency of aflatoxin contamination was particularly high. Japan is a country where many fermented products have been consumed, and fungus producing afla- 
toxin was isolated (Kurata, 1968). Other mycotoxins have been also studied (Saito, 1972), but it was not established that they have any meaning to frequent hepatocellular carcinoma in Japan.

Presented at the 28th Annual Meeting of Korean Society of Pathology in Seoul and at the satellite meeting of the 11th Meeting of Hepatology of Western Japan at Kurume in October, 1976.

\section{REFERENCES}

Alpert, M. E., Hutt, M. S. R., Wogan, G. N. and Davidson, C. S. (1969). Association between aflatoxin content of food and hepatoma frequency in Uganda. Cancer, 28, 253-260.

BeCKer, B.J.P. and Chatgidakis, C.B. (1961). Primary carcinoma of the liver in Johannesburg. Acta unio internat. canc., 17, 650653.

Berman, C. (1951). Primary carcinoma of the liver. A study in incidence, clinical manifestations, pathology and aetiology. H. K. Lewis and Co., London.

Borel, C. A. (1964). Contribution statistique a 1'etude anatomo-pathologique des cirrhosis hepatiques. Schweiz. Med. Wschr., 94, 1293-1297.

Börner, A. (19ô1). Aetiologie und Todesursache der verschiedenen Formen der Leberzirrhose in den Jahren 1955 bis 1959. Dtsch. Med. Wschr, 86, 43-47.

Caroli, J, Marchus, N. and Chevrel, B. (1970). Le cancer primitif du foie sur cirrhose. A propos de 120 cas. Med. Intern., 5, 917-934.

Dible, J. H. (1957). Fatty change, cirrhosis and liver cancer in Great Britain. Acta unio internat. canc., 13, 545-608.

Edmondson, H. A. and Steiner, P. E. (1954). Primary carcinoma of liver. Study of 100 cases among 48,900 necropsies. Cancer, 7, 462-503.

Elkington, S. G, McBrien,D.J. and Spencer, H. (1963). Hepatoma in cirrhosis. Brit. Med. J., 5371, 1501-1503.
Elsner, B. and JAUregui, E.M. (1974). Autopsy study of primary liver carcinoma in Buenos Aires, Argentina. Acta HepatoGstroenterol., 21, 26-34.

Epstein, S. (1964). Primary carcinoma of the liver. Am. J. Med. Sci., 247, 137-144.

ERvasti, J. (1967). Primary carcinoma of the liver. A pathologic and clinial study of 100 cases. Acta Chir. Scand. Suppl, 331, 1-65.

Gall, E. A. (1960 a). Primary and metastatic carcinoma of the liver. Relationship to hepatic cirrhosis. Arch. Path., 70, 226-232.

GALL, E. A. (1960 b). Posthepatitic, postnecrotic, and nutritional cirrhosis. A pathologic analysis. Am. J. Path., 36, 241-271.

Glennert, J. (1961). Primary carcinoma of the liver. A post-mortem study of 104 cases. Acta Path. Microbiol. Scand., 53, 50-60.

HALL, J.W. and Sun, S. C. (1951). Effect of portal cirrhosis on the development of carcinoma. Cancer, 4, 131-135.

HiRAi, H. (1975). Carcinofetal proteins. Colloq. Peptides Biol. Fluids, 23, 3-23.

Klasliwa, R. M. and Gupta, M. M. (1963). Evaluation of aetiological factors in cirrhosis of the liver as found in India. J. Indian Med. Assoc., 40, 406-413.

Kasliwa, R.M., Sharma,B.M. and Cathrvidi, G.C. (1965). Aetiological factors of cirrhosis of the liver in adults in India. A study of 290 cases. J. Indian Med. Assoc., 44, 407-413.

KnopP, J. (1961). Beurteilung der Zunahme von Lebercirrhosen in einer Sektionsstatistik, Virchow's Arch., 334, 285-300.

KöHN, K. (1956). Der primäre Leberkrebs. Springer, Berlin, 1955 ; Ueber das Vorkommen von Mikrocarcinomen in cirrhotischen Lebern. Z. Krebsforsch, 61, 350-363.

Koizumi, T., Kamata, T, Nuguishi, T., Nasu, A. Kamedaka, H., Goto, S., Botan, Y. and Matsubara, Y. (1971). Nutritional status of the patients with hepatocellular carcinoma. Relation between the development of hepatocellular carcinoma and nutritional condition. Kanzo (Acta Hepatol. Japan). $12,361-366$.

Kosaka, A. (1963). From hepatitis to cirrhosis. Proc. 16th Med. Congr. Japan, 3, 368-375.

Kurata, H. (1968). Aflatoxin producing fungi isolated from foodstuffs in Japan. J. Food Hyg. Soc. Japan, 9, 29-34. 
Leevy, C. M., Gellene, R. and Ning,M. (1964). Primary liver cancer in cirrhosis of the alcohlics. Ann. N.Y. Acad. Sci, 114, 10261040.

Lopez-Corella, E., Ridaura-Sanz, C. and AlBORes-SaAvedra, J. (1968). Primary carcinoma of the liver in Mexican adults. Cancer, 22, 678-685.

LUNZENAUER, K. (1955). Ueber das primäre Lebercarcinom. Betrachtungen an Hand von 26, 515 Sektionen aus den Jahren 1920-1954.

Z. Krebsforsch., 60, 630-638.

MacDonald, R. A. (1956). Cirrhosis and primary cancer of the liver: Changes in their occurrence at the Boston City Hospital 1897-1954. New Eng. J. Med., 255, 1179-1183.

Manderson, W.G., Patick, R.S. and Peters, E. E. (1965). Primary carcinoma of the liver. A survey of cases admitted to Glasgow Royal Infirmary 1949-1963. Scott. Med. J., 10, 60-64

Meyer, P.G. (1954). Das primäre Lebercarcinom in Basel. Z. Krebsforsch., 60, 115-138.

Miyai, K. and Ruebner, B. H. (1963). Acute yellow atrophy, cirrhosis, and hepatoma, their incidence at the Johns Hopkins Hospital 1917-1960. Arch. Path., 75, 609-617.

Miyaji, T. YU,K. Oda, T., Nagatomo,T. and Sawada, K. (1963). Pathological study of the primary carcinoma of the liver in Japan during recent 10 years. Kanzo (Acta $\mathrm{He}$ patol. Japon.), 1, 17-36.

MiYaJi, T. (1965). Primary carcinoma of the liver in Japan, specially on the association with cirrhosis. Trans. Japan. Path. Soc., 54, 23-38.

Miyaji, T. (1976). Association of hepatocellular carcinoma with cirrhosis among autopsy cases in Japan during 14 years from 1958 to 1971. GANN Monograph on Cancer Res., 18, 129-149.

Miyake, M. (1960). Pathology of the liver, particularly on cirrhosis. Trans. Japan. Path. Soc., 49, 589-592.

NaGayo, M. (1914). Referate Über Leberzirrhose (pathologisch-anatomischer Teil).

Trans. Japan. Path. Soc, 4, 31-72.

Nielsen, J.O. (1971). Incidence and meaning of persistence of Australia antigen in patients with acute viral hepatitis: development of chronic hepatitis. New Eng. J. Med., 285, 1157-1160.

NishiOKA, K. (1972). Association between he- patocellular carcinoma and Australia antigen. Naikagaku Zasshi (Japan. J. Clin. Med.) 30, 1154-1158.

Nishioka, K., Levin, A.G., and Simons, M.J. (1975): Hepatitis B antigen, antigen subtypes and Hepatitis B antibody in normal subjects and patients with liver disease. Bull. W. H. O., 52, 293-300.

Ohbayashi, A., Оhкоснi, K. and Mayumi, M. (1972). Familial clustering of asymptomatic carriers of Australia antigen and patients with chronic liver disease or primary liver cancer. Gastroenterol., 62, 618-625.

Ohbayashi, A. and Mayumi,M. (1974). HBAg subtypes in familial carriers. Lancet, i (7856), 507.

Ottolenghi, A. (1966). Rilievi statisctici su carcinomi primitive e cirrhos del fegato. Cancro, 19, 559-567.

PARKER, R.G.F. (1957). The incidence of primary hepatic carcinoma in cirrhosis. Proc. Roy. Soc. Med., 50, 145-147.

Patton, R.B. and Horn, R. C., Jr. (1964). Primary liver carcinoma. Autopsy study of 60 cases. Cancer, 17, 757-768.

Péquignot, H., Etienne, J. P., Delavierre, P. and Petite, J. P. (1967). Cancer primitifs $\mathrm{du}$ foie sur cirrhose. Augmentation de fréquence et observation chez des cirrhostiques connus et suivis. Press Méd., 75, 2595-2600.

Prince, A. M. (1970). S. H. antigen and chronic liver disease. Lancet, ii, 717-718.

Purtilo, D. T. and Gottlieb, L. S. (1973). Cirrhosis and hepatoma occurring at Boston City Hospital (1917-1968). Cancer, 32, 458-462.

Riva, G. and Weiner, D.M. (1960). Zur Aetiologie der Leberzirrhose. Statistische Erhebung an 331 autoptisch verifizierten Fällen von Leberzirrhose. Bull. Schweiz. Akad. Med. Wiss., 16, 65-77.

Roulet, F. C. (1957). Anatomo-pathological consideration on primitive cancer of the liver. Acta unio internat. canc., 13, 623-627.

Sagebiel, R.W., McFarland, R. B. and Taft, E. B. (1963). Primary carcinoma of the liver in relation to cirrhosis. Am. J. Clin. Path., 40, 516-520.

SaIto, M. (1972). Pathological study of mycotoxin-in pursuit of the lesion caused by mycotoxin as an index. Trans. Japan. Path. Soc., 61, 33-70.

SAKURAI, M. (1969). A histopathologic study 
on the effect of alcohol on cirrhosis and hepatoma of autopsy cases in Japan. Acta Path. Japon, 19, 283-314.

San Jose, D., Cady, A., West, M., Chomet, B. and Zimmerman, H. J. (1965). Primary carcinoma of the liver. Analysis of clinical and biochemical features of 80 cases. Am. J. Digest. Dis., 10, 657-674.

SARAGOCA, A. (1964). Primary neoplasms iof the liver. The possibility of biochemical diagnosis. Am. J. Digest. Dis., 9, 337-344.

Schmitz, A. (1971). Incidence des cirrhoses et des hépatomes dans un material d'autopsies. Evolution au cours des ving dernières anńees. Gastro-enterol. Belg., 34, 523-535.

Scrinivasa, R. K. and Sharma, R. D. (1968). Type of cirrhosis as an aetiological agent in primary carcinoma of the liver. Indian J. Path. Bacteriol, 11, 8-12.

Segi, M. and Kurihara, M. (1972). Cancer mortality for selected sites in 24 countries. Japan Cancer Society, 6, 1966-1967.

Shanmugaratnam, K. (1961). Liver cancer and cirrhosis in Singapore. Acta unio internat. canc., 17, 898-902.

Sievers, B. U. (1973). Leberzirrhose und Leberkarzinom in 10 Jahren einer Prosektur. Acta Hepato-Gastroenterol, 20, 483-790.
Sundarsanam, D., Kutumbiah, P, Samuel, J. and Gault, E.W. (1963). Primary carcinoma of the liver. A study of 25 cases proved at postmortem examination. Indian $\mathrm{J}$. Path. Bacteriol., 6, 8-18.

TikTinskiı, O. L. (1961). Primary cancer of the liver. Sov. Med, 9, 107-109.

Tung, G., Cheng, K.H. and Hou, S. E. (1964).A study on the morphology and etiology of Laennec's cirrhosis. Chin. Med. J., 83, 143154.

Voigt, K. G. and Helbig, W. (1968). Zur Pathologie des primären Leberkarzinoms. Obduktionsbefunde von 124 Fällen. Arch. Geschwulstforsch., 31, 39-71.

Wainwright, J. (1961). Cirrhosis in the African in Natal. Acta unio internat. canc., 17, 677-679.

Wöckel,W. and Altrock,H. (1968). Zur Häufigkeit der Leberzirrhose und des Leberkrebses in Obduktionsgut. Dtsch. Gesundh. Wiss., 23, 165-169.

YING, Y. Y., MA, C. C., HsÜ, Y. T., LEI, H. H., LiAnG, S. F., Liv, C. H. and KU, C. Y. (1963). Primary carcinoma of liver with special reference to histogenesis and its relationship to liver cirrhosis. Chin. Med. J., 82, 279-294. 